\title{
A SPECTRAL APPROACH TO STATISTICAL POLAR SHAPE MODELING
}

\author{
Jia $\mathrm{Li}$ \\ Alfred O. Hero \\ Department of Electrical Engineering and Computer Science \\ University of Michigan \\ 1301 Beal Avenue, Ann Arbor, MI 48109 USA
}

\begin{abstract}
Accounting for uncertainty in three-dimensional (3D) shapes is important in a large number of scientific and engineering areas including: biometrics, biomedical imaging, and multimodality image registration. It is well known that 3D star-shaped objects can be represented by Fourier descriptors such as spherical harmonics and double Fourier series. However, the statistics of these spectral shape models have not been widely explored. This article presents a spectral theory and its applications in 3D shape modeling. Spherical harmonic (SH) expansions over the unit sphere not only provide a low dimensional polarimetric parameterization of stochastic shape, but also correspond to Karhunen-Loéve (K-L) expansion of any isotropic random field on the unit sphere. Spherical harmonic expansions permit estimation and detection tasks, such as optimal shape filtering, object registration, and shape classification, which can be performed directly in the spectral domain with low computational complexity.
\end{abstract}

\section{INTRODUCTION}

Techniques of three dimensional shape modeling have been widely studied over the past two decades [1]. In many computer vision related areas, such as pattern recognition, deformation and motion analysis, image registration and image retrieval, shape modeling techniques have been integrated with other techniques to achieve different goals. In this paper, "shape modeling" refers to surface boundary representations of 3D polar objects. Deterministic surface descriptions, such as polygons, B-splines and Fourier descriptors, have been well established [1]. Among these descriptions, the parametric representations which are object-centered and use a linear combination of basis functions, are of special interest to us. These mappings from spatial object space to parameter space provide a compact representation of the object and are useful for shape storage, noise filtering, and pattern recognition. Although deterministic models are successfully employed in many applications, they are incapable of reflecting the variations within a class of shapes. In medical imaging, for instance, anatomical shape can change significantly during a treatment. It is highly desirable to have reliable statistical shape models which can characterize typical ranges of shape variation and capture meaningful statistical information. Such information can be used to develop optimal algorithms for noise removal, object registration and segmentation, and establish tight bounds on achievable performance.

Many researchers have explored the area of statistical shape modeling [2, 3, 4]. The common procedure of these approaches

This work was partly supported by the National Cancer Institute, United States Department of Health and Human Services under Grant R01 CA87955. is as follows. First, extract shape features or shape parameters from training data sets. These features may include labeled "landmark" points [2]; coefficients of Fourier series model [3]; and distance map [4]. Next, compute the mean and variance of the shape or shape parameters from the features extracted in the first step. Usually principle component analysis (PCA) is used to compute variance and characterize typical variations of the shape. Finally, the statistical properties of the shape are incorporated into a image processing algorithm to accomplish registration and segmentation. Our approach is related to Staib's deformable model [3] since we also use Fourier series as basis functions to model 3D shapes. We propose an isotropic random field model for 3D polar shape objects using spherical harmonics as the eigen-functions of Karhunen-Loéve expansion. The novelty of our approach lies in treating the coarse segmentation result as a random field over the unit sphere and using the spectral theory of random fields over the sphere to obtain statistically uncorrelated shape parameters. We apply the developed statistical shape model to two problems in computer vision. The first one is optimal shape filtering: given noisy samples of surface boundary points, e.g. coarsely segmented from an object, find an optimal estimate of the true surface boundary. The second problem is the $3 \mathrm{D}$ object registration problem. Based on Burel's method [5] and a statistical noise model, we propose a maximum likelihood estimator which can simultaneously estimate the spherical harmonic coefficients and register 3D objects at different orientations.

\section{STATISTICAL POLAR SHAPE MODELING}

\subsection{Fourier Descriptors}

The surface of a polar shaped object can be represented by its radial coordinate $r$ with respect to a selected origin inside the object. Here $r$ is a single valued function of $\theta$ and $\phi$, where $(\theta, \phi)$ is a direction vector on the unit sphere $S^{2}$, i.e. $r: S^{2} \rightarrow \mathbb{R}$. (Instead of $(\theta, \phi)$, we sometimes use single variables, such as $s$ and $t$, to represent direction vectors in $S^{2}$.) The unit sphere $S^{2}$ is defined as the sphere of radius 1 and centered at origin. Fourier descriptors represent polar shaped objects as a linear combination of orthonormal basis functions. Spherical harmonics $\left\{Y_{l}^{m}(\theta, \phi)\right\}$ are special functions defined on the unit sphere as

$$
Y_{l}^{m}(\theta, \phi)=(-1)^{m} \sqrt{\frac{2 l+1}{4 \pi} \frac{(l-m) !}{(l+m) !}} P_{l}^{m}(\cos \theta) e^{i m \phi}
$$

where $\theta \in[0, \pi]$ is the polar angle, $\phi \in[0,2 \pi]$ is the azimuthal angle, $P_{l}^{m}(x)$ is the associated Legendre function, $l$ is a non-negative integer, $m$ is an integer in $[-l, l]$. Spherical harmonics have been 
widely used to model 3D objects [6,3] because they are orthonormal, complete over the space of polar surfaces and ordered in spatial frequency. However, the statistical properties of this spectral model have not been widely explored.

\subsection{Random Field on Unit Sphere}

In the computer vision community, 2D rectangular random field models have been applied to texture synthesis, texture classification, and image segmentation. In shape modeling, it is reasonable to assume that radial functions of coarsely segmented objects are samples of random fields over the unit sphere $S^{2}$. However, to the best of our knowledge, no random field model has been reported for 3D shape modeling. We guess it is partly because of the difficulty to characterize an arbitrary random field over $S^{2}$, which is defined as follows.

Definition 1 A second order random field over $S^{2} \subset \mathbb{R}^{3}$ is a function $Z: S^{2} \rightarrow L_{2}(\Omega, \mathcal{F}, P)$, where $\Omega$ is a sample space with generic element $\omega, \mathcal{F}$ is a $\sigma$-algebra of subsets of $\Omega$, and $P$ is a probability measure on $\mathcal{F}$.

To reduce the complexity of the correlation function of $Z(\theta, \phi)$, we consider characterizing the shape of polar objects by an isotropic random field over $S^{2}$.

\subsection{Isotropic Random Field on $S^{2}$ and its orthogonal repre- sentation}

Let $\left(\theta_{1}, \phi_{1}\right)$ and $\left(\theta_{2}, \phi_{2}\right)$ denote two directions separated by angle $\gamma$ in the spherical coordinate system, as shown in Fig. 1. These angles satisfy the following trigonometric identity,

$$
\cos \gamma=\cos \theta_{1} \cos \theta_{2}+\sin \theta_{1} \sin \theta_{2} \cos \left(\phi_{1}-\phi_{2}\right) .
$$

The value $\cos \gamma$ is called the angular distance between the two directions $\left(\theta_{1}, \phi_{1}\right)$ and $\left(\theta_{2}, \phi_{2}\right)$.

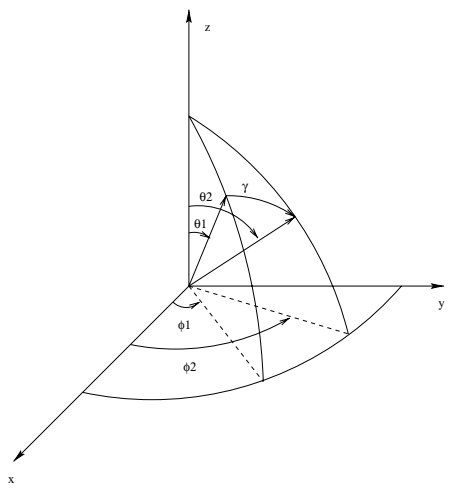

Fig. 1. Two directions in $S^{2},\left(\theta_{1}, \phi_{1}\right)$ and $\left(\theta_{2}, \phi_{2}\right)$, and the angle $\gamma$ between them.

Definition 2 A random field $X(\theta, \phi)$ on the unit sphere $S^{2}$ is called isotropic in the wide sense if its mean is constant

$$
E\{X(\theta, \phi)\}=\text { constant }
$$

and its correlation depends only on the angular distance $\cos \gamma$ defined in (2)

$$
E\left\{X\left(\theta_{1}, \phi_{1}\right) X^{*}\left(\theta_{2}, \phi_{2}\right)\right\} \triangleq R(\gamma)=\psi(\cos \gamma)
$$

The correlation function of such a random field can be thought as invariant to any rotation $g \in S O(3)$. Here $S O(3)$ denotes the group of rotations around the origin in $\mathbb{R}^{3}$. Isotropic random field models have been widely studied in many research areas, such as earth science, astrophysics and electrical field theory. In fact, this statistical property is satisfied by a large class of 3D shapes. For example, in biological shape analysis, the orientation of virus particles in the electron microscope can be completely disordered [7] and the radial function segmented from such a case forms an isotropic random field.

Theorem 1 Let $z: S^{2} \rightarrow \mathbb{R}$ be the radial function of a polar object which center has been aligned with the origin $O$ of the coordinate system. If the object center is fixed at $O$ and the orientation of the object is uniformly distributed, i.e. there is no preferred direction, the observed radial function $Z$ is an isotropic random field. It's mean and correlation function are determined by

$$
E[Z(t)]=\text { constant }=\frac{1}{4 \pi} \int_{x \in S^{2}} z(x) d \Omega_{x}
$$

and

$$
\begin{aligned}
& E[Z(s) Z(t)]=R_{Z}(\measuredangle(s, t)) \\
& =\frac{\int_{x \in S^{2}} \int_{\{y: \angle(x, y)=\angle(s, t)\}} z(x) z(y) d \Omega_{y} d \Omega_{x}}{4 \pi \cdot(2 \pi \sin \measuredangle(s, t))} .
\end{aligned}
$$

If $s=t$, (6) will be in the form of $E\left[Z^{2}(t)\right]=\frac{1}{4 \pi} \int_{x \in S^{2}} z^{2}(x) d \Omega_{x}$.

Proof:

Let $g$ be a random rotation operator in $S O(3)$ which has uniform distribution over $S O(3)$. The observed radial function $F$ can be expressed as $F(x)=g f(x)$. Since $g$ is uniformly distributed in $S O(3)$, the probability that any surface point falls in any sector $(\theta, \phi) \in S^{2}$ of area $\Delta \Omega_{S^{2}}$ is the same and equals $\frac{\Delta \Omega_{S^{2}}}{4 \pi}$. Taking the limit as $\Delta \Omega_{S^{2}} \rightarrow 0$ yields equation (5). Similarly, for two arbitrary directions $s$ and $t$ on the unit sphere, any pair of surface points which has the same angular distance as $\cos (\measuredangle(s, t))$ will be equally likely to be found in the directions of $s$ and $t$. Therefore equation (6) gives the proper correlation function of $F$. End of proof.

In [8], Yadrenko pointed out that any isotropic random field over the unit sphere can be orthogonally decomposed by spherical harmonics.

Theorem 2 ([8]) A mean-square continuous isotropic random field $X(\theta, \phi)$ of zero mean in $S^{2}$ can be represented as:

$$
X(\theta, \phi)=\sum_{l=0}^{\infty} \sum_{m=-l}^{l} A_{l}^{m} Y_{l}^{m}(\theta, \phi)
$$

with $Y_{l}^{m}(\theta, \phi)$ denoting the spherical harmonics of degree $l$ and order $m$, and

$$
A_{l}^{m}=\int_{\theta=0}^{\pi} \int_{\phi=0}^{2 \pi} X(\theta, \phi) Y_{l}^{m *}(\theta, \phi) \sin \theta d \theta d \phi
$$

such that $E\left\{A_{l}^{m}\right\}=0$ and $E\left\{A_{l}^{m} A_{l^{\prime}}^{* m^{\prime}}\right\}=\lambda_{l} \delta_{l, l^{\prime}} \delta_{m, m^{\prime}}$ where $\lambda_{l}=2 \pi \int_{-1}^{1} \psi(t) P_{l}(t) d t$ is the coefficient in the Legendre series of the correlation function, and $\psi(\cos \gamma)=R(\gamma)$ is the correlation function of $X(\theta, \phi)$.

Based on this spectral theorem, the coefficients in the SH expansion of an isotropic Gaussian random field will be independent random variables for different $l$ and $m$. This important property will be used in two applications in the next section. 


\section{APPLICATIONS OF STATISTICAL SHAPE MODELING}

\subsection{Optimal Shape filtering on $S^{2}$}

Relations 7 and 8 imply that spherical harmonics are eigenfunctions in the Karhunen-Loéve expansion of an isotropic random field over the unit sphere $S^{2}$ [8]. It is well known that Wiener filtering can be implemented in the original or K-L domains. Based on the spectral theory of random fields and the spherical geometry of polar objects, one can also in principle use this theory to decompose the radial function and estimate uncorrelated noisy shape parameters. The detailed procedure is described in the text following.

Let $F(x): S^{2} \rightarrow(0,+\infty)$ represent the radial function of a polar object acquired through some segmentation process. It is assumed that $\bar{F}=E[F]$ and that the zero mean random field $F-$ $\bar{F}$ can be decomposed as:

$$
F(x)-\bar{F}(x)=S(x)+W(x)
$$

where $S$ is an isotropic zero mean random field and $W$ represents an independent white Gaussian noise field. The correlation function of $S$ can be represented by $R_{S}(x, y)=R_{S}(\measuredangle(x, y))$. Strictly speaking, for consistency $S$ and $W$ must be such that $S+W \geq-\bar{F}$ w.p.1. We will sidestep this issue by assuming that the standard deviations of $S$ and $W$ are much smaller than $\bar{F}$. By relations 7 and 8, the K-L expansion of $S$ is a linear combination of spherical harmonics,

$$
S(x)=\sum_{l=0}^{\infty} \sum_{m=-l}^{l} a_{l}^{m} Y_{l}^{m}(x)
$$

where $a_{l}^{m}$ is independent random variable (for all $l, m$ ) with zero mean and variance $E\left[a_{l}^{m} a_{l^{\prime}}^{m^{\prime}}\right]=\lambda_{l} \delta_{l, l^{\prime}} \delta_{m, m^{\prime}}$, where $\lambda_{l}$ is determined by $\lambda_{l}=2 \pi \int_{-1}^{1} R_{S}(x) P_{l}(x) d x$. Let $\sigma_{W}^{2}$ be the variance of the white Gaussian noise term in 9. The optimal estimator of the shape parameter $a_{l}^{m}$ is the conditional mean $E\left[a_{l}^{m} \mid F_{l}^{m}\right]$ which can be written as:

$$
\hat{a}_{l}^{m}=\frac{\int_{S^{2}}(F-\bar{F}) Y_{l}^{m}(x) d \Omega_{S^{2}} \cdot \lambda_{l}}{\lambda_{l}+\sigma_{W}^{2}} .
$$

The optimal estimator of $S$ is a linear combination of spherical harmonics weighted by $\hat{a}_{l}^{m}$ :

$$
\hat{S}(x)=\sum_{l=0}^{\infty} \sum_{m=-l}^{l} \hat{a}_{l}^{m} Y_{l}^{m}(x)
$$

\subsection{Joint Shape and Orientation Estimation of 3D Objects}

Finding the rotation of a 3D object is a common problem. Considering the registration of $3 \mathrm{D}$ objects in different orientations, Burel [5] proposed to use $\mathrm{SH}$ as orthogonal basis to decompose the 3D shapes and get invariants for object recognition. Assuming an isotropic Gaussian noise model, we develop a maximum likelihood (ML) method to jointly estimate the spherical harmonic coefficients and the Euler angles of 3D rotation based on Burel's method.

Any rotation $g$ in $S O(3)$ can be completely determined by Euler angles $\alpha, \beta, \gamma$. In terms of group theory, spherical harmonics expand an irreducible representation space of the rotation $g$ [9] Let the radial function of a 3D object have a representation:

$$
R(\theta, \phi)=\sum_{l=0}^{K} \sum_{m=-l}^{l} c_{l}^{m} Y_{l}^{m}(\theta, \phi) .
$$

for some positive $K$. Applying the 3D rotation operator $g$ to the object gives a new radial function $\tilde{R}(\theta, \phi)=g R(\theta, \phi)$, which can be written as

$$
\tilde{R}(\theta, \phi)=\sum_{l=0}^{K} \sum_{m=-l}^{l} \tilde{c}_{l}^{m} Y_{l}^{m}(\theta, \phi) .
$$

The spherical harmonic coefficients $\tilde{c}_{l}^{m}$ in (14) and $c_{l}^{m}$ in (13) have the following relationship:

$$
\tilde{c}_{l}^{m}=\sum_{m^{\prime}=-l}^{l} D_{m m^{\prime}}^{l}(\alpha, \beta, \gamma) c_{l}^{m^{\prime}}
$$

where,

$$
D_{m m^{\prime}}^{l}(\alpha, \beta, \gamma)=\exp (-i m \alpha) \cdot d_{m m^{\prime}}^{l}(\beta) \cdot \exp \left(-i m^{\prime} \gamma\right)
$$

and

$$
\begin{aligned}
& d_{m m^{\prime}}^{l}(\beta) \\
= & (-1)^{l-m^{\prime}} \sqrt{(l+m) !(l-m) !\left(l+m^{\prime}\right) !\left(l-m^{\prime}\right) !} \\
& \times \sum_{k}(-1)^{k} \frac{\left(\cos \frac{\beta}{2}\right)^{m+m^{\prime}+2 k}\left(\sin \frac{\beta}{2}\right)^{2 l-m-m^{\prime}-2 k}}{k !(l-m-k) !\left(l-m^{\prime}-k\right) !\left(m+m^{\prime}+k\right) !} .
\end{aligned}
$$

In (17), the summation is carried out over all values of $k$ producing positive integers under the factorial symbol.

Under the assumption that the segmentation noise is an isotropic Gaussian random field over $S^{2}$, the spherical harmonic coefficients before and after rotation can be modeled as: $c_{l}^{m}=a_{l}^{m}+\eta_{l}^{m}$ and $\tilde{c}_{l}^{m}=\sum_{n=-l}^{l} D_{m n}^{l}(\alpha, \beta, \gamma) a_{l}^{n}+\tilde{\eta}_{l}^{m}$, where $\left\{a_{l}^{m}, l=\right.$ $1 \cdots K, m=-l \cdots l\}$ is the set of true SH coefficients describing the 3D shape before rotation, and $\eta_{l}^{m}$ and $\tilde{\eta}_{l}^{m}$ are the zero mean Gaussian noise with variance $\sigma_{l}^{2}$ and $\tilde{\sigma}_{l}^{2}$. By Theorem 2 in Section 2.2, $\eta_{l}^{m}$ and $\tilde{\eta}_{l}^{m}$ are independent Gaussian random variables for different $l$ and $m$. The variances $\sigma_{l}^{2}$ and $\tilde{\sigma}_{l}^{2}$ are determined by: $\sigma_{l}^{2}=2 \pi \int_{-1}^{1} \psi(t) P_{l}(t) d t$ and $\tilde{\sigma}_{l}^{2}=2 \pi \int_{-1}^{1} \tilde{\psi}(t) P_{l}(t) d t$, where $\psi(t)$ and $\tilde{\psi}(t)$ are the correlation functions of the respective isotropic noise fields and $P_{l}(t)$ is a Legendre polynomial.

Therefore, the likelihood functions for $c_{l}^{m}$ and $\tilde{c}_{l}^{m}$ are:

$$
L_{c_{l}^{m}}=\exp \left(-\frac{\left(c_{l}^{m}-a_{l}^{m}\right)^{2}}{2 \sigma_{l}^{2}}\right)
$$

and

$$
L_{\tilde{c}_{l}^{m}}=\exp \left(-\frac{\left(\tilde{c}_{l}^{m}-\sum_{n=-l}^{l} D_{m n}^{l}(\alpha, \beta, \gamma) a_{l}^{n}\right)^{2}}{2 \tilde{\sigma}_{l}^{2}}\right) .
$$

Using the fact $\eta_{l}^{m}$ and $\tilde{\eta}_{l}^{m}$ are independent for different $l$ and $m$, we propose to jointly estimate $\alpha, \beta, \gamma$, and $\left\{a_{l}^{m}\right\}$ via maximum likelihood:

$$
\begin{aligned}
\hat{\alpha}, \hat{\beta}, \hat{\gamma},\left\{\hat{a}_{l}^{m}\right\}= & \underset{\alpha, \beta, \gamma,\left\{a_{l}^{m}\right\}}{\operatorname{argmin}} \sum_{l=1}^{K} \sum_{m=-l}^{l}\left(\frac{\left(c_{l}^{m}-a_{l}^{m}\right)^{2}}{2 \sigma_{l}^{2}}\right. \\
& \left.+\frac{\left(\tilde{c}_{l}^{m}-\sum_{n=-l}^{l} D_{m n}^{l}(\alpha, \beta, \gamma) a_{l}^{n}\right)^{2}}{2 \tilde{\sigma}_{l}^{2}}\right) .
\end{aligned}
$$


Note that the maximum likelihood estimate is equivalent to a weighted least squares fitting problem, which is a nonlinear optimization here. As is the case with many such implicitly defined estimators, the minimum can not be found analytically and iterative minimization of the objective should be employed.

\section{EXPERIMENT RESULTS}

\subsection{Wiener Filter Shape Denoising}

In this experiment, we simulated radial functions of 3D objects, which are sampled from an isotropic random field over $S^{2}$ with additive white Gaussian noise. To evaluate the performance of the proposed Wiener filtering shape denoising, we compare it with the performance of average filtering, which was implemented through a convolution of the noisy shape with an average filter. Fig. 2 plots the results of Wiener filtering and average filtering of the same random field. Fig. 2(a) shows the bias of Wiener filtering and average filtering results. The rough surface represents the bias of the average filtering result, while the darker surface which is relatively flat represents the bias of the Wiener filtering result. In Fig. 2(b), the variances of the two filtering results are plotted. It can be seen that the output of Wiener filtering (darker surface) has a much smaller variance than the output of average filtering.

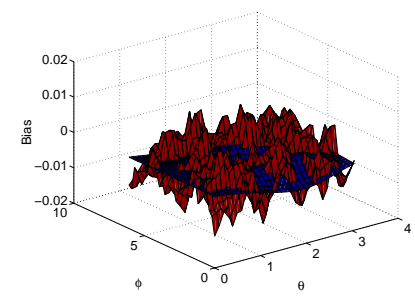

(a) Bias

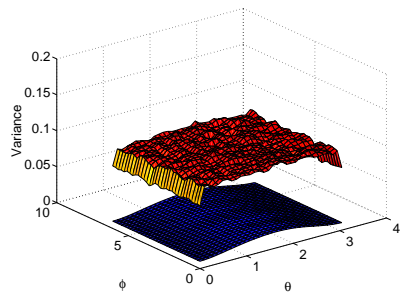

(b) Variance
Fig. 2. Comparison of Wiener filtering and linear filtering of isotropic random field on $S^{2}$.

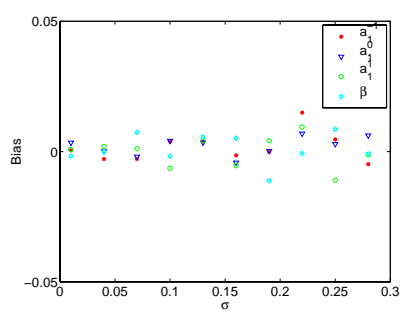

(a) Bias

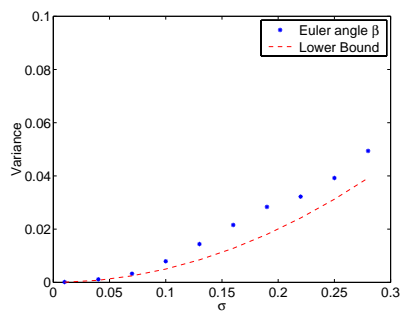

(b) Variance of $\hat{\beta}$
Fig. 3. The performance of joint estimators and the Cramér-Rao bound.

\subsection{Joint 3D Registration and Shape Estimation}

In the second experiment, the proposed maximum likelihood method is implemented to jointly estimate the $3 \mathrm{D}$ rotation and spherical harmonic coefficients of the noise contaminated objects. The estimators' biases are plotted versus the standard deviation of the Gaussian noise in Fig.3(a). From the observation, we can see that the estimator is virtually unbiased. In Fig.3(b), the variance of the maximum likelihood rotation angle estimator $\hat{\beta}$ is compared to the derived Cramér-Rao lower bound. It can be seen that the standard deviation of the estimation error is less than the standard deviation of the noise process. Therefore, the joint estimation has improved the estimation performance for the spherical harmonic coefficients. Since the boundary information in the two sets of images is correlated, this is an expected result. The performance of the rotation angle estimator is also close to the lower bound, which shows that the proposed estimator is nearly an efficient estimator.

\section{CONCLUSIONS}

We proposed an isotropic random field model for random 3D shapes. This model characterizes the shape information by a correlation function of the random field, which admits a K-L expansion in terms of spherical harmonics. We applied such a statistical model to the problems of optimal shape filtering and object registration. The results show that Wiener filtering over $S^{2}$ has a much lower variance than average filtering. We developed a maximum likelihood method to jointly estimate 3D rotation angles and $\mathrm{SH}$ shape parameters. Since the $3 \mathrm{D}$ objects are registered in the frequency domain via low order SH coefficients, the registration automatically filters out high frequency noise and has low computational complexity.

\section{REFERENCES}

[1] R. M. Bolle and B. C. Vemuri, "On three-dimensional surface reconstruction methods," IEEE Trans. on Pattern Analysis and Machine Intelligence, vol. 13, no. 1, pp. 1-13, Jan. 1991.

[2] T. F. Cootes, C. J. Taylor, D. H. Cooper, and J. Graham, "Active shape models - their training and application," Computer Vision and Image Understanding, vol. 61, no. 1, pp. 38-59, Jan. 1995.

[3] L. H. Staib and J. S. Duncan, "Model-based deformable surface finding for medical images," IEEE Transactions on Pattern Analysis and Machine Intelligence, vol. 15, no. 5, pp. 1996, Oct. 1996.

[4] M. E. Leventon, W. E. L. Grimson, and O. Faugeras, "Statistical shape influence in geodesic active contours," in Proceedings IEEE Conference on Computer Vision and Pattern Recognition, 2000, vol. 1, pp. 316-323.

[5] G. Burel, "Three-dimensional invariants and their application to object recognition," Signal Processing, vol. 45, pp. 1-22, 1995.

[6] P. Haigron, G. Lefaix, X. Riot, and R. Collorec, "Application of spherical harmonics to the modelling of anatomical shapes," Journal of Computing and Information Technology, vol. 6, no. 4, pp. 449-461, December 1998.

[7] P. C. Doerschuk and J. E. Johnson, "Ab inito reconstruction and experimental design for cryo electron microscopy," IEEE Trans. on Information Theory, vol. 46, no. 5, pp. 1714-1729, Aug. 2000.

[8] M. I. Yadrenko, Spectral theory of random fields, Optimization Software, 1983.

[9] E. Wigner, Group Theory and its Application to Quantum Mechanics of Atomic Spectra, Academic Press, 1959. 